\title{
Promoter DNA methylation and expression levels of HOXA4, HOXA5 and MEIS1 in acute myeloid leukemia
}

\author{
EWA MUSIALIK $^{1 *}$, MATEUSZ BUJKO ${ }^{1 *}$, PAULINA KOBER ${ }^{1}$, MONIKA ANNA GRYGOROWICZ $^{2}$, \\ MARTA LIBURA ${ }^{3}$, MARTA PRZESTRZELSKA ${ }^{3}$, PRZEMYSŁAW JUSZCZYŃSKI ${ }^{4}$, KATARZYNA BORG $^{4}$, \\ IZABELA FLOREK $^{5}$, MAŁGORZATA JAKÓBCZYK ${ }^{5}$ and JANUSZ ALEKSANDER SIEDLECKI ${ }^{1}$ \\ Departments of ${ }^{1}$ Molecular and Translational Oncology and ${ }^{2}$ Immunology, \\ Maria Sklodowska-Curie Memorial Cancer Center and Institute of Oncology, Warsaw 02-781; \\ ${ }^{3}$ Department of Hematology, Oncology and Internal Diseases, The Medical University of Warsaw, Warsaw 02-097; \\ ${ }^{4}$ Department of Diagnostic Hematology, Institute of Hematology and Transfusion Medicine, Warsaw 02-109; \\ ${ }^{5}$ Department of Hematology, Jagiellonian University, Cracow 31-501, Poland
}

Received February 14, 2014; Accepted November 3, 2014

DOI: $10.3892 / \mathrm{mmr} .2015 .3196$

\begin{abstract}
H O X A$ genes encode transcription factors, which are crucial for embryogenesis and tissue differentiation and are involved in the early stages of hematopoiesis. Aberrations in HOXA genes and their cofactor MEISI are found in human neoplasms, including acute myeloid leukemia (AML). The present study investigated the role of HOXA4, HOXA5 and MEIS1 promoter DNA methylation and mRNA expression in AML. Samples from 78 AML patients and 12 normal bone marrow (BM) samples were included. The levels of promoter DNA methylation were determined using quantitative methylation-specific polymerase chain reaction (PCR; qMSP) and the relative expression levels were measured using reverse transcription quantitative PCR in Ficoll-separated BM mononuclear cells and in fluorescent activated cell sorting-sorted populations of normal hematopoietic progenitors. In total, 38.1 and $28.9 \%$ of the patients exhibited high methylation levels of HOXA4 and HOXA5, respectively, compared with the control samples, and MEIS1 methylation was almost absent. An inverse correlation between HOXA4 methylation and expression was identified in a group of patients with a normal karyotype (NK AML). An association between the genes was observed and correlation between the DNA methylation and expression levels of the HOXA gene promoter with the expression of MEISI was observed. Patients
\end{abstract}

Correspondence to: Mr. Mateusz Bujko, Department of Molecular and Translational Oncology, Maria Sklodowska-Curie Memorial Cancer Center and Institute of Oncology, W.K. Roentgena 5, Warsaw 02-781, Poland

E-mail: mbujko@coi.waw.pl

*Contributed equally

Key words: HOXA4, HOXA5, MEIS1, DNA methylation, expression level, acute myeloid leukemia with favorable chromosomal aberrations revealed a low level of HOXA4 methylation and decreased expression levels of HOXA5 and MEIS1 compared with the NK AML and the adverse cytogenetic risk patients. The NK AML patients with NPM1 mutations exhibited elevated HOXA4 methylation and expression levels of HOXA5 and MEIS1 compared with the NPM1 wild-type patients. Comparison of the undifferentiated $\mathrm{BM}$-derived hematopoietic $\mathrm{CD} 34^{+} \mathrm{CD} 38^{\text {low }}, \mathrm{CD} 34^{+} \mathrm{CD} 38^{+}$and $\mathrm{CD}_{15}{ }^{+}$cells revealed a gradual decrease in the expression levels of these three genes and an increase in HOXA4 promoter methylation. This differentiation-associated variability was not observed in AML, which was classified according to the French-American-British system.

\section{Introduction}

Acute myelogenous leukemia (AML) is a heterogeneous disease caused by the uncontrolled proliferation of myeloid precursor cells with abnormal maturation.

Genes encoding transcription factors (TF), involved in normal hematopoiesis, are among the most common targets of genetic aberrations in AML (1). This involves chromosomal translocations, including the most prevalent $\mathrm{t}(8 ; 21)$ (RUNX1-ETO), inv (16) (CBF-MYH11) and 11q23 MLL rearrangements and point mutations in key regulators of hematopoietic cell differentiation, termed class II mutations. Among these point mutations, the most frequent are changes in CEBPA, NPM1, RUNX1 and $M L L$ (2). Recurrent chromosomal translocations, together with CEBPA and NPM1 mutations, constitute the basis of the current World Health Organization (WHO) classification and are included in the guidance for AML patients risk stratification (3).

In addition to genetic alterations, epigenetic aberrations are also involved in leukemogenesis. DNA methylation is one of the most widely described epigenetic elements involved in regulating gene expression. Aberrant DNA methylation in the regulatory regions of the genes encoding hematopoietic TFs has been observed in AML patients, including early-acting TFs, such as HOX cluster genes (4). 
The present study focused on the role of DNA methylation and the expression levels of three selected early-acting hematopoietic TFs genes: HOXA4, HOXA5 and MEIS1 in AML.

HOXA genes are clustered at the 7 p15.2 chromosomal region and these encode TFs that are crucial for embryogenesis and tissues differentiation (5). HOXA proteins are also involved in the early stages of hematopoiesis and lineage specification (6).

HOXA4 controls hematopoietic stem cell (HSC) self-renewal and the expansion of either myeloid- and lymphoid-primed progenitors (7). In addition to its paralogs, HOXB4 and HOXD4, HOXA4 is also involved in the inhibition of cell differentiation (8).

Another HOXA cluster gene, HOXA5, governs the specification of myeloid and erythroid lineages. Its constitutive expression inhibits erythropoiesis and promotes myelopoiesis $(9,10)$. MEIS1 is a transcription activator-like effector protein, which functions as a HOX protein cofactor. This protein exerts its function by enhancing HOXA4 and HOXA5 DNA binding specificity (11).

Aberrations in the HOXA and MEISI genes have been identified in human neoplasms, including AML (5). The HOXA4, HOXA5 and MEIS1 genes are frequently hypermethylated in different types of leukemia, including adult AML $(4,12)$. Previous studies have demonstrated the prognostic value of HOXA4 and HOXA5 methylation in leukemia patients $(4,13)$.

\section{Materials and methods}

Patients. Bone marrow (BM) samples from 78 AML patients were used and $12 \mathrm{BM}$ samples were obtained from healthy donors as controls. The patients were classified based on their French-American-British (FAB) subtypes and their cytogenetic statuses. The BM mononuclear cells (BMMC) were isolated from the BM samples using Ficoll density gradient centrifugation at $400 \mathrm{x}$ g for $30 \mathrm{~min}$ at room temperature. The patient characteristics are shown in Table I. The present study was approved by the committee of M. Sklodowska-Curie Memorial Cancer Center and Institute of Oncology (Warsaw, Poland).

Cell sorting. The $\mathrm{CD}_{3} 4^{+}$progenitor cells were purified from normal BMMC samples using a Dynabeads ${ }^{\circledR}$ CD34 Positive Isolation kit (Invitrogen Life Technologies, Carlsbad, CA, USA). The CD $34^{+}$fraction was subsequently labeled with fluorescein isothiocyanate (FITC)-conjugated anti-CD34 and APC-conjugated anti-CD38 antibodies. The depleted fraction (CD34-) was labeled with FITC-conjugated anti-CD15 antibodies (all antibodies were purchased from BD Biosciences, Franklin Lakes, NJ, USA). The CD $34^{+} \mathrm{CD} 38^{-}, \mathrm{CD} 34^{+} \mathrm{CD} 38^{+}$and $\mathrm{CD} 15^{+} \mathrm{BM}$ cell populations were sorted using a BD FACSAria (BD Biosciences). The results are presented in Fig. 1.

Quantitative DNA methylation analysis. The DNA was extracted from the BMMC using a QIAamp DNA Mini kit (Qiagen, Hilden, Germany) and subsequently subjected to bisulfite conversion using an EpiTect kit (Qiagen). The cells isolated by fluorescence-activated cell-sorting were subjected to direct bisulfite conversion using an EpiTect Lyse All kit (Qiagen). The levels of DNA methylation were determined by
Table I. Characteristics of patients with acute myeloid leukemia in the present study.

\begin{tabular}{|c|c|}
\hline Patient characteristic & Number of patients ${ }^{b}$ \\
\hline \multicolumn{2}{|l|}{ Gender } \\
\hline Male & $38 / 78$ \\
\hline Female & $40 / 78$ \\
\hline \multicolumn{2}{|l|}{ Age (years) } \\
\hline$<60$ & $64 / 77$ \\
\hline$\geq 60$ & $13 / 77$ \\
\hline \multicolumn{2}{|l|}{ FAB classification } \\
\hline M0 & $6 / 76$ \\
\hline M1 & $16 / 76$ \\
\hline M2 & $16 / 76$ \\
\hline M3 & $10 / 76$ \\
\hline M4 & $15 / 76$ \\
\hline M5 & $13 / 76$ \\
\hline \multicolumn{2}{|l|}{ Cytogenetics } \\
\hline Inv (16) & $7 / 76$ \\
\hline $\mathrm{T}(8 ; 21)$ & $9 / 76$ \\
\hline $\mathrm{T}(15 ; 17)$ & $8 / 76$ \\
\hline 3q/11q abnormalities & $6 / 76$ \\
\hline Complex karyotype & $4 / 76$ \\
\hline Normal karyotype & $31 / 76$ \\
\hline Other & $11 / 76$ \\
\hline \multicolumn{2}{|l|}{ Cytogenetic risk } \\
\hline Favorable & $24 / 76$ \\
\hline Intermediate & $33 / 76$ \\
\hline Adverse & $19 / 76$ \\
\hline \multicolumn{2}{|l|}{$C E B P A$ mutations $^{\mathrm{a}}$} \\
\hline Negative & $25 / 29$ \\
\hline Positive & $4 / 29$ \\
\hline \multicolumn{2}{|l|}{ NPM1 mutations ${ }^{\mathrm{a}}$} \\
\hline Negative & $12 / 29$ \\
\hline Positive & $17 / 29$ \\
\hline \multicolumn{2}{|l|}{ FLT3-Itd } \\
\hline Negative & $57 / 76$ \\
\hline Positive & $19 / 76$ \\
\hline \multicolumn{2}{|l|}{$M L L-P t d$} \\
\hline Negative & $56 / 61$ \\
\hline Positive & $5 / 61$ \\
\hline \multicolumn{2}{|c|}{ WBC count at diagnosis $\left(10^{3} / \mathrm{Ml}\right)$} \\
\hline Median & 23,15 \\
\hline Range & $0.3-331.1$ \\
\hline \multicolumn{2}{|c|}{ Blast percentage in bone marrow } \\
\hline Median & 75 \\
\hline Range & $6.3-98$ \\
\hline
\end{tabular}

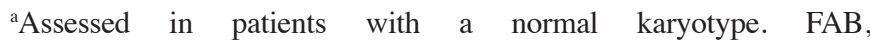
French-American-British; WBC, white blood cell. ${ }^{b}$ Number of patients with a trait/total number of patients with a given characteristic assessed.

quantitative methylation-specific PCR (qMSP). Each region of interest was amplified with methylation-specific primer pairs using reverse transcription (RT) qPCR in parallel to a reference $A C T B$ region containing no $\mathrm{CpG}$ dinucleotides. The $\mathrm{RT}-\mathrm{qPCR}$ 
Table II. Sequences of polymerase chain reaction primers and probes used for quantitate methylation-specific analysis.

\begin{tabular}{llll}
\hline Gene & \multicolumn{1}{c}{ Forward } & \multicolumn{1}{c}{ Reverse } & \multicolumn{1}{c}{ Probe } \\
\hline HOXA4 & 5'-GTAGTATTTATT & 5'-CCGTACCCC & 5'Fam-CCCCACCAATAA \\
& ACGTATTCGCGC-3' & ACGTACAACG-3' & ACGCACCGCG-Tamra-3' \\
HOXA5 & 5'-AATGGGTTGTAA & 5'-CGTTCAACC & 5'Fam-AAAACAAAACTC \\
& TTTTAATTCGATTTC-3' & GAACTCGAACG-3' & ATCGCCCAACTTCCG-Tamra-3' \\
\multirow{2}{*}{ MEIS1 } & 5'-TGCGGTTAG & 5'-CATAACAAATCG & 5'-CATTAAACTACAACAAAT \\
& AGTTCGTTTCGC-3' & CGTCTTACACAA-3' & AAACTCCTCGAC-Tamra-3' \\
ACTB & 5'-TGGTGATGGAGG & 5'-AACCAATAAAAC & 5'Fam-ACCACCACCCAACAC \\
& AGGTTTAGTAAGT-3' & CTACTCCTCCCTTAA-3' & ACAATAACAAACACA-Tamra-3' \\
\hline
\end{tabular}

reaction was performed in $7.5 \mu \mathrm{l}(1.5 \mu \mathrm{l}$ bisulfite-converted DNA template, 2X SensiMix II Probe mastermix (Bioline, London, $\mathrm{UK}$ ), $2.25 \mathrm{pmol}$ of each primer (4.5 pmol of each primer for $A C T B)$ and 1.88 pmol of the probe. The RT-qPCR amplification was performed using an Applied Biosystems 7900 HT Sequence Detection system (Applied Biosystems, Foster City, CA, USA) in 384-well plates. The sequences of the primers and probes used are shown in Table II.

The standard curves of known DNA template concentrations were used to quantify the resulting PCR product. These were prepared using serial dilutions of plasmid DNA containing PCR product inserts of each amplified region. The plasmid constructs were obtained by amplification of standard methylated genomic DNA (Qiagen), with the use of each primer pair and cloning of the PCR products using a Strataclone kit (Agilent, Santa Clara, CA, USA). The plasmid DNA was amplified in bacteria, purified using a Plasmid Mini kit (A\&A Biotechnology, Gdynia, Poland) and quantified using Quanti-iT Picogreen (Invitrogen Life Technologies, Carlsbad, CA, USA).

The levels of DNA methylation, determined by the percentage of the methylated reference (PMR), was calculated by dividing the 'gene of interest':ACTB ratio of a patient sample by the 'gene of interest':ACTB ratio of the methylated DNA in the control sample (Qiagen) and multiplying by 100 .

An EpiTect Control DNA and Control DNA set (Qiagen) containing human methylated and unmethylated DNA served as positive and negative control samples, respectively.

Expression analysis. RNA was isolated from the BMMC using an RNeasyMini kit (Qiagen) and quantified using a NanoDrop 2000 Spectrophotometer (ThermoScientific, Waltham,MA, USA). Each RNA sample (500 ng) was subjected to RT using SuperScript II Reverse Transcriptase (Invitrogen Life Technologies). The RNA from the cells, sorted by flow cytometry, was extracted using RNAqueous-Micro (Ambion Life Technologies, Carlsbad, CA, USA) and subjected to RT, as previously.

The expression levels of HOXA4, HOXA5 and MEISI were assessed using gene expression assays: Hs00269972_s1, Hs00270931_s1 and Hs00357657_m1 (Applied Biosystems). The ubiquitin gene assay (Hs00824723_m1) (Applied Biosystems) was used as a reference. RT-qPCR was performed using the following cycling conditions: $95^{\circ} \mathrm{C}$ for $10 \mathrm{~min}$ followed by 45 cycles of $95^{\circ} \mathrm{C}$ for $15 \mathrm{sec}$ and $60^{\circ} \mathrm{C}$ for $60 \mathrm{sec}$. Gene expression was calculated using the $2^{-\Delta \mathrm{Ct}}$ method.
Statistical analysis. The gene expression levels and DNA methylation levels (PMR) were analyzed using a two-sided Mann-Whitney U-test, Kruskal-Walis test and Spearman's correlation. For descriptive statistics, the quantitative methylation results were also categorized into binary data. The samples were classified as methylation-positive/methylation-high or methylation-low based on the assessment of DNA methylation level in the normal BM samples. The mean value \pm two standard deviations of the BM results was used as a threshold for each gene independently. A two-sided exact Fisher's test was used for the comparison of proportions. $\mathrm{P}<0.05$ was considered to indicate a statistically significant difference. The statistical evaluation and visualization was performed using GraphPad Prism software 5.03 (GraphPad, La Jolla, CA, USA).

\section{Results}

Promoter DNA methylation and gene expression levels in AML patients and controls. The levels of DNA methylation were assessed using qMSP in 78 AML patient samples and 12 normal BM samples. The promoter regions of HOXA4 and HOXA5 revealed variable degrees of methylation in the patient-derived samples and the normal BM cells. AML samples with a methylation level exceeding the threshold value, described previously, were classified as methylation-high. According to these criteria, high levels of HOXA4 and HOXA5 promoter DNA methylation were observed in 38.1 (30/76) and 28.9\% (21/76), respectively, in the AML patients.

A degree of HOXA4 and HOXA5 promoter methylation in the three sorted hematopoietic progenitors populations was also observed. The level of HOXA4 methylation was higher in the immature $\mathrm{BM}$-derived $\mathrm{CD} 15^{+}$granulocytes compared with the early $\mathrm{CD} 34^{+}$progenitors. The HOXA5 methylation level, however, did not vary between the sorted progenitor fractions, but was higher compared with the total BMMC. DNA methylation was not observed in the MEISI promoter in either the patient or normal samples. The results are shown in Fig. 1C.

The RNA extraction and assessment of relative gene expression levels were successfully performed in 70 patient and $8 \mathrm{BM}$ samples. All three genes of interest exhibited homogenous, low levels of expression in the normal BM samples. Marked heterogeneity was observed in the AML patient expression levels of HOXA4, HOXA5 and MEIS1, with a notable proportion of patients having high expression levels compared to the BM cells (Fig. 1A-C). 

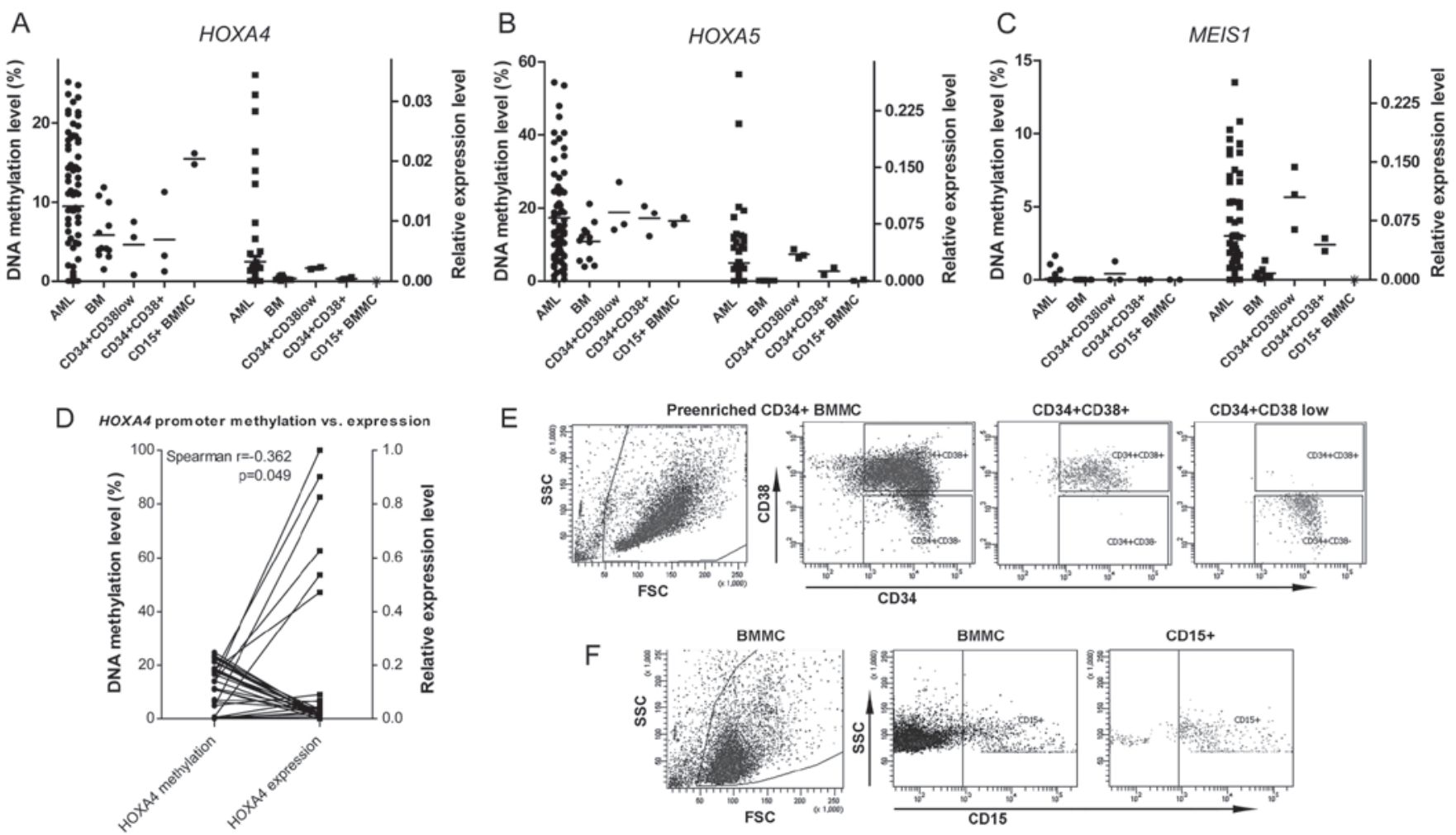

Figure 1. Comparison between the promoter DNA methylation and the relative expression levels of (A) HOXA4, (B) HOXA5 and (C) MEIS1 in AML and normal $\mathrm{BM}$ and $\mathrm{CD} 34^{+} \mathrm{CD} 38^{\text {low }}, \mathrm{CD} 34^{+} \mathrm{CD} 38^{+}$and $\mathrm{CD} 15^{+}$cells isolated from normal BMMC. The horizontal lines represent the mean value. (D) Correlation of HOXA4 promoter DNA methylation and expression levels in the NK AML. Representative example of pre- and post-sort analysis of (E) normal BM $\mathrm{CD} 34^{+} \mathrm{CD} 38^{\text {low }}, \mathrm{CD} 34^{+} \mathrm{CD} 38^{+}$and $(\mathrm{F}) \mathrm{CD} 15^{+}$cells. The cell populations were sorted using BD FACSAria (BD Bioscience). AML, acute myeloid leukemia; BM, bone marrow; BMMC, bone marrow mononuclear cells; NK AML, normal karyotype acute myeloid leukemia; SSC, side scatter; FSC, forward scatter.

The three examined genes were differentially expressed when the hematopoietic progenitors and immature $\mathrm{CD} 15^{+}$cells were compared. The highest level of expression was observed in the earliest $\mathrm{CD} 34^{+} \mathrm{CD} 38^{\text {low }}$ progenitors, the expression level decreased in the more mature $\mathrm{CD} 34^{+} \mathrm{CD} 38^{+}$cells and expression either further decreased or was lost in the CD15+ cells (Fig. 1E). The decreased expression of HOXA4 in the three sorted populations corresponded to an increase of gene promoter DNA methylation.

Correlation between the HOXA4 and HOXA5 promoter DNA methylation levels was observed in AML (Spearman $\mathrm{r}=0.3020 ; \mathrm{P}=0.0080$ ). Correlation was also observed between the expression levels of HOXA4 and HOXA5 and the expression of their cofactor MEISl (Spearman $\mathrm{r}=0.3182 ; \mathrm{P}=0.0068$ and Spearman $\mathrm{r}=0.800 ; \mathrm{P}<0.0001$, respectively).

The analysis of the association between promoter methylation and expression levels in the entire group of AML patients revealed no statistically significant correlation. This was also preformed separately in cytogenetically normal AML patients and an inverse correlation was observed between the expression of HOXA4 and the levels of gene methylation (Spearman $r=-0.362 ; P=0.049$; Fig. 1D).

Gene promoter DNA methylation, expression levels and cytogenetic risk. The DNA methylation and expression levels were compared in the AML patients, grouped according to their cytogenetic status, which constitutes the basis of the current WHO classification and risk assessment.
The patients in the favorable prognostic group demonstrated a lower level of HOXA4 methylation compared with those in the intermediate and high risk groups. The NK patients and those carrying unfavorable translocations exhibited the highest variability in levels of HOXA4 methylation (Fig. 2A). No significant differences were detected in the levels of HOXA5 methylation between the patients with distinct cytogenetic risk or status.

The gene expression analysis revealed differences in the expression levels of HOXA5 and MEISI between patients with different cytogenetic statuses (Fig. 2B and C). All the patients carrying the favorable translocations, $t(8 ; 21)$, inv (16) and $\mathrm{t}(15 ; 17)$, exhibited low expression levels of HOXA5 and MEISI and the expression levels of HOXA5 and MEIS1 were generally lower in the favorable prognostic group compared with the intermediate and high risk patients. Notably, all the patients with $3 q$ aberrations revealed low expression levels of the two genes compared with the favorable risk patients. However, the $3 \mathrm{q}$ group consisted of only three patients.

No significant variation was observed in the HOXA4 expression level between distinct cytogenetic risk and status groups.

Promoter DNA methylation/expression levels in patients grouped according to FAB. The FAB classification system has been commonly used to classify AML patients on the basis of leukemic cell morphology, cytochemistry and maturation (14). In the present study, the patients were grouped into 
A

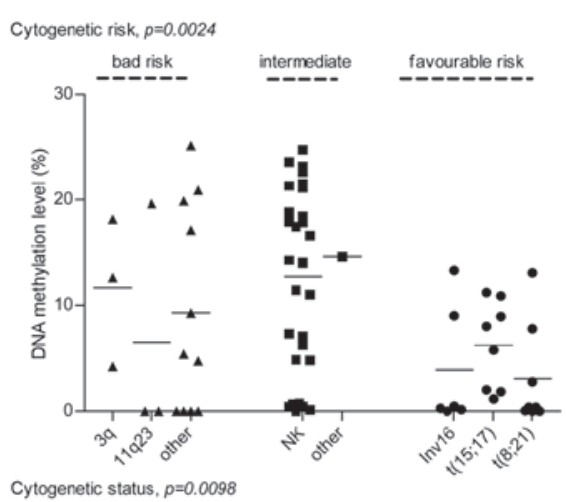

B

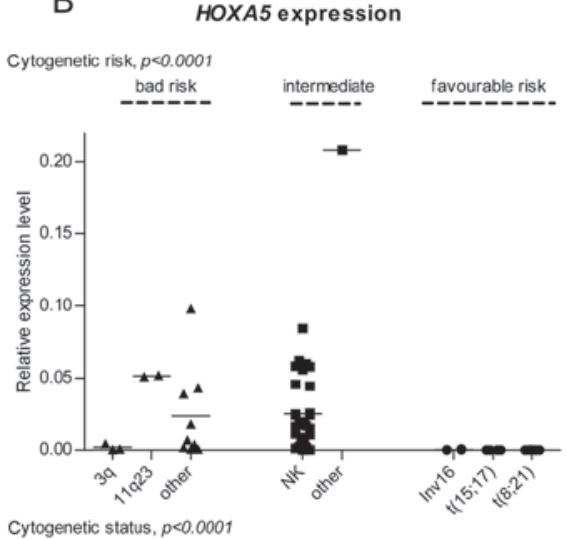

C

MEIS1 expression

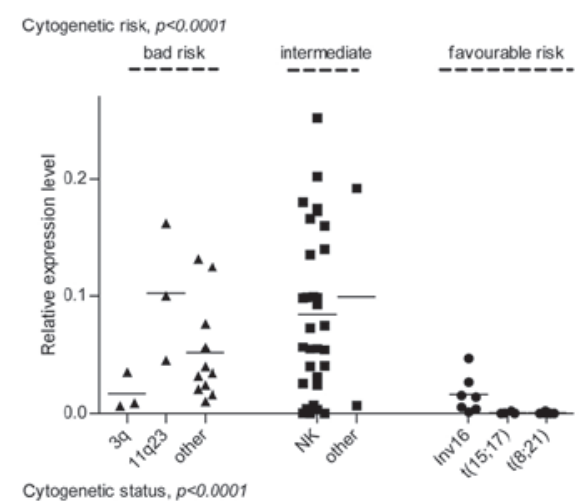

Figure 2. Comparison between the promoter methylation levels of (A) HOXA4 and relative expression levels of (B) HOXA5 and (C) MEIS1 in AML, stratified according to cytogenetic status. Horizontal lines represent the mean. AML, acute myeloid leukemia; NK, normal karyotype.
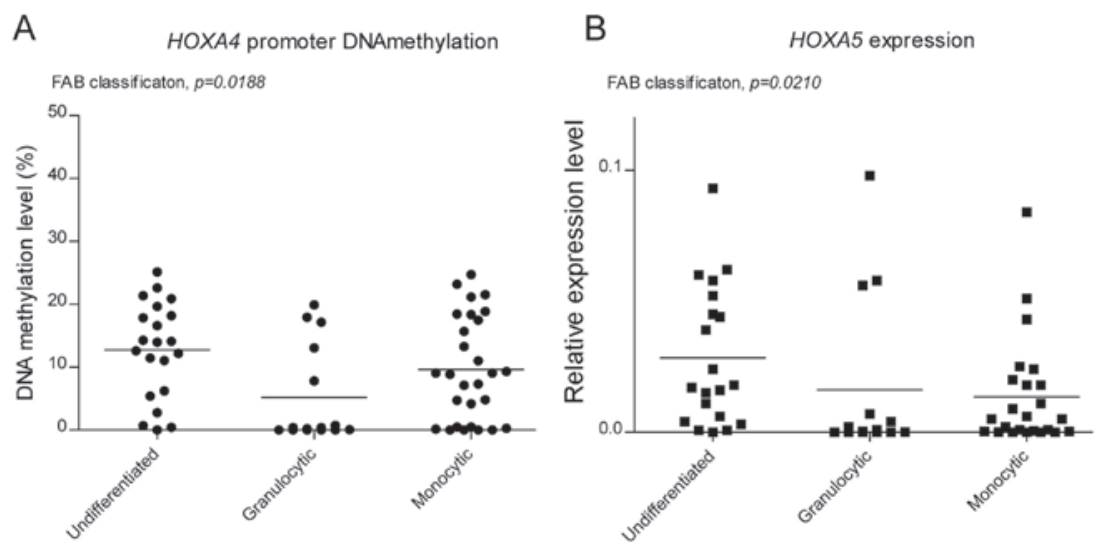

Figure 3. Differences in the (A) promoter methylation level of HOXA4 and (B) relative expression level of HOXA5 in AML, classified according to FAB into three categories: AML without differentiation, AML with granulocytic and AML with monocytic maturation. Horizontal lines represent the mean. AML, acute myeloid leukemia; FAB, French-American-British.

three categories based on the FAB classification system: AML without maturation (M0 and M1), AML with granulocytic maturation (M2) and AML with monocytic maturation (M4 and M5). These three groups were then compared in terms of HOXA4, HOXA5 and MEIS1 promoter methylation and expression levels. The M3 PML patients were excluded as they constitute a separate cytogenetic group defined by the presence of $\mathrm{t}(15: 17)$, which is described above. The groups differed significantly in $H O X A 4$ promoter methylation and expression levels of HOXA5 (Fig. 3A and B, respectively). The HOXA4 DNA methylation level was lowest in the granulocytic AMLs and highest in the AMLs without maturation. This differed to the previous observations in the normal hematopoietic progenitors (Fig. 1A), where the undifferentiated CD34 ${ }^{+}$ cells exhibited lower HOXA4 methylation compared with the immature granulocytes $\left(\mathrm{BMMC} \mathrm{CD} 15^{+}\right)$. The patients with granulocytic characteristics revealed the lowest expression levels of HOXA5, whereas the highest mean value was observed in patients without maturation.

In AML without maturation, high expression levels of HOXA5 were observed compared with the AML with granulocytic differentiation, which was concordant with the observed difference between the isolated $\mathrm{CD} 34^{+}$progenitor and the BMMC CD15 ${ }^{+}$immature granulocytes (Fig. 1B).
DNA promoter methylation and $m R N A$ expression analysis in the AML patients according to the FLT3-ITD and NPM1 mutation status in NK AML. The promoter methylation and expression levels of HOXA4, HOXA5 and MEIS1 were compared in the entire group of AML patients, stratified according to FLT3-ITD, which is a poor prognostic factor. The patients with FLT3-ITD (FLT3-ITD+) demonstrated significantly elevated expression levels of HOXA5 and MEIS1 compared with those without mutation with a 3.57-fold $(\mathrm{P}=0.0043)$ and 2.38 -fold $(\mathrm{P}=0.0048)$ change in mean relative expression, repectively. No differences were observed in DNA promoter methylation levels depending on the presence of FLT3-ITD.

Mutations in NPMI and CEBPA are favorable prognostic factors, which are used for the risk assessment in AML patients without recurrent chromosomal abnormalities (2). The present study compared the methylation and expression levels of the three genes of interest in NK AML, stratified according to NPM1 mutation status. The results revealed elevated HOXA4 methylation (2.03-fold change in the mean DNA methylation level; $\mathrm{P}=0.0178$ ) and elevated expression levels of HOXA5 and MEISI in patients carrying the NPM1 mutation (NPM1+) (3.27-fold change; $\mathrm{P}=0.0007$ and 2.38-fold change; $\mathrm{P}=0.0048$, respectively). Since $C E B P A$ mutations were identified in only four AML patients, statistical evaluation was not performed. 


\section{Discussion}

HOXA transcription factors and their cofactor MEIS1 are among the key regulators of development and differentiation. They are involved in the initial stages of hematopoiesis and contribute to subsequent lineage specification (8-10). Aberrant HOXA expression is associated with numerous types of cancer and DNA hypermethylation has been identified as a mechanism partially responsible for the downregulation of these genes (5).

The present study focused on the role of promoter DNA methylation and expression levels of HOXA4, HOXA5 and MEIS1 in AML.

HOXA4 and HOXA5 promoters have been previously described to be frequently methylated in AML (4). Analysis of these genes in the present study revealed promoter methylation in normal BM samples (mean 4.1 and $11.5 \%$ for $\mathrm{HOXA4}$ and HOXA5, respectively). Therefore a cutoff value for methylation-high samples was applied based on the results for the normal BM. The frequency of HOXA4 and HOXA5 hypermethylation observed in the present study differed from previous studies, possibly resulting from the use of different analytical techniques and threshold levels for 'methylated' sample classification. HOXA4 hypermethylation was previously reported to occur in 64\% (by combined bisulfite restriction analysis; COBRA) (4) and $77 \%$ (by methylation-sensitive melting curve analysis) (12) of patients compared with $39.4 \%$ of patients in the present study. HOXA5 hypermethylation in AML was previously reported as $36 \%$ (13) by pyrosequencing and 60 and $59 \%$ by COBRA $(4,15)$, compared to $27.6 \%$ in the present study. A slight correlation between the DNA methylation levels of HOXA4 and HOXA5 were observed in the AML samples in the present study, which may reflect the fact that the two HOXA genes are located closely within the same chromosomal region and are transcriptionally coregulated. This association was also observed in childhood leukemia (4). Previously reported results, indicating that MEISI is hypermethylated in $15 \%$ of AML patients and frequently methylated in patients with $\mathrm{t}(8: 21)$ (16) were not observed in the present study.

Gene expression is regulated through epigenetic mechanisms, which include DNA methylation. It has been proposed that HOXA4 downregulation may be associated with promoter methylation status, which was supported by observations in chronic lymphoblastic leukemia (17) and AML (12). HOXA5 promoter methylation regulates gene expression in myeloid leukemia cell lines (15).

AML is a heterogenous and complex disease, in which chromosomal aberrations are important in determining the leukemia biology and prognosis (3). The biological diversity of AML is the predominant factor, which may explain the lack of correlation between the promoter methylation and the expression levels of HOXA4 and HOXA5 in the entire group of patients. This association was additionally analyzed in NK AML and an inverse correlation of HOXA4 methylation and expression was observed. The comparison of $\mathrm{CD} 34^{+} \mathrm{CD} 38^{\text {low }}, \mathrm{CD} 34^{+} \mathrm{CD} 38^{+}$ and $\mathrm{CD} 15^{+} \mathrm{BM}$ derived cells also supported the involvement of HOXA4 promoter methylation in regulating gene expression, as an increase in DNA methylation levels corresponded to a gradual decrease of RNA expression.

The role of HOXA4 expression in myeloid leukemogenesis has been investigated previously. AML patients with low expression levels of $\mathrm{HOX} A 4$ have a poor prognosis in terms of overall survival $(18,19)$, however, a previous study identified HOXA4 among the genes overexpressed in patients with poor outcome (20). The prognostic role is possibly more complex as it also dependent on the expression of MEIS1 (12).

The role of low expression levels of HOXA4 as an adverse prognostic factor has been observed in a subgroup of cytogenetically normal patients (21) and also on an entire group of AML patients (18), despite the fact that low levels of HOXA4 occur in patients with favorable translocations, particularly $\mathrm{t}(15: 17)(22,23)$. The poor survival rates observed in AML with low HOXA4 expression in a study by Tholouli et al (18) was possibly due to the high representation of NK AML patients and the fact that this group of patients is characterized by a high and heterogenous level of gene expression $(18,21,24,25)$. The expression of HOXA4 may therefore be involved, particularly in molecularly heterogenous cytogenetically normal patients, for which new prognostic markers are required.

In the present study, no statistically significant difference was observed in the expression of HOXA4 between the AML cytogenetic groups, however different levels of promoter methylation were observed in this gene. A high, but variable HOXA4 methylation level was observed in patients with NK AML and those with adverse cytogenetic risk compared with patients a with favorable prognosis. This was concordant with the previously reported differences in the frequency of HOXA4 hypermethylation between the cytogenetic risk groups (12). Considering the observed association between HOXA4 promoter methylation and expression levels in NK AML and the prognostic role of the expression of this gene in AML, it was suggested that the DNA methylation level of this gene may have a prognostic value. This is consistent with previously reported data, in which HOXA4 was among the genes upregulated in NK AML patients with the NPM1 mutation, a favorable prognostic factor $(23,26)$. However, this is inconsistent with the observation in the present study of a higher HOXA4 methylation level in a group of NK AML patients with the NPM1 mutation.

Different levels of HOXA4 promoter DNA methylation were observed when groups of patients, stratified according to the FAB classification system, were compared. However, by contrast to the results in normal hematopoietic precursors, the highest methylation level was observed in undifferentiated AMLs. Aberrant HOXA4 methylation in leukemic BM samples may be associated with the neoplastic nature of leukemic cells rather than their differentiation stage.

HOXA5 promoter methylation was previously identified as an independent prognostic factor in a group of AML patients (13). As this study involved a small number of patients, the observed prognostic role was considered a possible result of higher levels of HOXA 5 methylation in the favorable cytogenetic group. In the present study, no difference in the methylation levels between the prognostic groups of patients was observed, which indirectly supported the previous finding. In the AML patients, no association was found between the HOXA5 methylation and expression levels, as observed in myeloid leukemia cell lines (15).

Unlike HOXA5 promoter methylation, diverse gene expression levels were observed when patients with different molecular profiles were analyzed. Similar to HOXA4 promoter methylation, the expression levels of either HOXA5 or MEISI were lowest in the cytogenetically favorable patients and the 
FLT3 wild-type patients, as reported previously (25). Notably, in NK AML, the two genes were expressed at higher levels in the patients with NPM1 mutations, as has been observed in genome-wide expression profiling $(27,28)$. This suggested that the HOXA and MEISI genes may have slightly different roles in NK AML compared with AML patients with cytogenetic abberations.

Elevated expression levels of the three genes were observed in AML patients, compared with normal BM patients. The HOXA and MEIS1 genes were expressed at relatively high levels in the primitive $\mathrm{CD} 34^{+} \mathrm{CD} 38^{\text {low }}$ cells, decreased in the $\mathrm{CD} 34^{+} \mathrm{CD} 38^{+}$ progenitors and were further decreased or absent in the $\mathrm{BM}$ derived $\mathrm{CD}_{1} 5^{+}$cells. It is possible that the expression levels of the HOXA genes reflect the differentiation stage of leukemic blasts, however the regulation of these genes appeared to be generally impaired in AML. The expression levels of HOXA4 and HOXA5 may exceed their expression levels in normal cells, including normal early progenitors. The phenotypic features of the AML samples, according to the FAB classification, were not associated with HOXA or MEISl methylation or expression, with the exception of higher expression levels of HOXA5 in AML without differentiation compared with AML with granulocytic and monocytic differentiation. Similar observations have been reported previously, in which M1 patients revealed higher expression levels of HOXA5 compared with other AML patients (29).

It appears that there is an association between HOXA4, HOXA5 and MEISI in AML. The two investigated HOXA genes are closely associated. They are located in the same gene locus and appear to be transcriptionally coregulated with MEISl. The present study observed a correlation between the promoter methylation and gene expression of the HOXA genes and also observed a correlation between their expression and that of MEISI. This association was explicit between HOXA5 and MEISI and the two genes exhibited corresponding mRNA levels in the distinct molecular groups of the AML patients.

\section{Acknowledgements}

The present study was supported by the Ministry of Science and Higher Education of Poland (no. 344/N-INCA/2008/0).

\section{References}

1. Shima $\mathrm{Y}$ and Kitabayashi I: Deregulated transcription factors in leukemia. Int J Hematol 94: 134-141, 2011.

2. Döhner K and Döhner H: Molecular characterization of acute myeloid leukemia. Haematologica 93: 976-982, 2008.

3. Vardiman J and Hyjek E: World health organization classification, evaluation, and genetics of the myeloproliferative neoplasm variants. Hematology Am Soc Hematol Educ Program 2011: 250-256, 2011.

4. Strathdee G, Holyoake TL, Sim A, et al: Inactivation of HOXA genes by hypermethylation in myeloid and lymphoid malignancy is frequent and associated with poor prognosis. Clin Cancer Res 13: 5048-5055, 2007.

5. Shah N and Sukumar S: The Hox genes and their roles in oncogenesis. Nat Rev Cancer 10: 361-371, 2010.

6. Argiropoulos B and Humphries RK: Hox genes in hematopoiesis and leukemogenesis. Oncogene 26: 6766-6776, 2007.

7. Fournier M, Lebert-Ghali CÉ, Krosl G and Bijl JJ: HOXA4 induces expansion of hematopoietic stem cells in vitro and confers enhancement of pro-B-cells in vivo. Stem Cells Dev 21: 133-142, 2012.

8. Iacovino M, Hernandez C, Xu Z, Bajwa G, Prather M and Kyba M: A conserved role for Hox paralog group 4 in regulation of hematopoietic progenitors. Stem Cells Dev 18: 783-792, 2009.
9. Fuller JF, McAdara J, Yaron Y, Sakaguchi M, Fraser JK and Gasson JC: Characterization of HOX gene expression during myelopoiesis: role of HOX A5 in lineage commitment and maturation. Blood 93: 3391-3400, 1999.

10. Crooks GM, Fuller J, Petersen D, Izadi P, Malik P, Pattengale PK, et al: Constitutive HOXA5 expression inhibits erythropoiesis and increases myelopoiesis from human hematopoietic progenitors. Blood 94: 519-528, 1999.

11. Argiropoulos B, Yung E and Humphries RK: Unraveling the crucial roles of Meis1 in leukemogenesis and normal hematopoiesis. Genes Dev 21: 2845-2849, 2007.

12. Zangenberg M, Grubach L, Aggerholm A, Silkjaer T, Juhl-Christensen C, Nyvold CG, et al: The combined expression of HOXA4 and MEIS1 is an independent prognostic factor in patients with AML. Eur J Haematol 83: 439-448, 2009.

13. Kim SY, Hwang S-H, Song EJ, Shin HJ, Jung JS and Lee EY: Level of HOXA5 hypermethylation in acute myeloid leukemia is associated with short-term outcome. Korean J Lab Med 30: 469-473, 2010.

14. Walker H, Smith FJ and Betts DR: Cytogenetics in acute myeloid leukaemia. Blood Rev 8: 30-36, 1994.

15. Strathdee G, Sim A, Soutar R, Holyoake TL and Brown R: HOXA5 is targeted by cell-type-specific $\mathrm{CpG}$ island methylation in normal cells and during the development of acute myeloid leukaemia. Carcinogenesis 28: 299-309, 2007.

16. Lasa A, Carnicer MJ, Aventín A, Estivill C, Brunet S, Sierra J, et al: MEIS 1 expression is downregulated through promoter hypermethylation in AML1-ETO acute myeloid leukemias. Leukemia 18: 1231-1237, 2004.

17. Strathdee G, Sim A, Parker A, Oscier D and Brown R: Promoter hypermethylation silences expression of the Hox A4 gene and correlates with IgVh mutational status in CLL. Leukemia 20: 1326-1329, 2006

18. Tholouli E, MacDermott S, Hoyland J, Yin JL and Byers R: Quantitative multiplex quantum dot in-situ hybridisation based gene expression profiling in tissue microarrays identifies prognostic genes in acute myeloid leukaemia. Biochem Biophys Res Commun 425: 333-339, 2012.

19. Grubach L, Juhl-Christensen C, Rethmeier A, et al: Gene expression profiling of Polycomb, Hox and Meis genes in patients with acute myeloid leukaemia. Eur J Haematol 81: 112-122, 2008.

20. Bullinger L, Döhner K, Bair E, et al: Use of gene-expression profiling to identify prognostic subclasses in adult acute myeloid leukemia. N Engl J Med 350: 1605-1616, 2004.

21. Grubach L, Juhl-Christensen C, Rethmeier A, et al: Gene expression profiling of Polycomb, Hox and Meis genes in patients with acute myeloid leukaemia. Eur J Haematol 81: 112-122, 2008.

22. Thompson A, Quinn MF, Grimwade D, et al: Global down-regulation of HOX gene expression in PML-RARalpha+acute promyelocytic leukemia identified by small-array real-time PCR. Blood 101: 1558-1565, 2003.

23. Andreeff M, Ruvolo V, Gadgil S, et al: HOX expression patterns identify a common signature for favorable AML. Leukemia 22: 2041-2047, 2008

24. Debernardi S, Lillington DM, Chaplin T, et al: Genome-wide analysis of acute myeloid leukemia with normal karyotype reveals a unique pattern of homeobox gene expression distinct from those with translocation-mediated fusion events. Genes Chromosomes Cancer 37: 149-158, 2003.

25. Roche J, Zeng C, Barón A, et al: Hox expression in AML identifies a distinct subset of patients with intermediate cytogenetics. Leukemia 18: 1059-1063, 2004

26. Verhaak RG, Goudswaard CS, van Putten W, et al: Mutations in nucleophosmin (NPM1) in acute myeloid leukemia (AML): association with other gene abnormalities and previously established gene expression signatures and their favorable prognostic significance. Blood 106: 3747-3754, 2005.

27. Bacher U, Kohlmann A, Haferlach C and Haferlach T: Gene expression profiling in acute myeloid leukaemia (AML). Best Pract Res Clin Haematol 22: 169-180, 2009.

28. Whitman SP, Maharry K, Radmacher MD, et al: FLT3 internal tandem duplication associates with adverse outcome and geneand microRNA-expression signatures in patients 60 years of age or older with primary cytogenetically normal acute myeloid leukemia: a Cancer and Leukemia Group B study. Blood 116: 3622-3626, 2010.

29. Drabkin HA, Parsy C, Ferguson K, Guilhot F, et al: Quantitative HOX expression in chromosomally defined subsets of acute myelogenous leukemia. Leukemia 16:186-195, 2002. 